\title{
A Technique for Sensing Organic Compounds Using Fluorescence Maximum Shift In Laser Dyes
}

Jagdish W Dadge ${ }^{1 *}$ and Thakur $\mathrm{D}^{2}$

${ }^{1}$ Department of Physics, College of Engineering, Shivajinagar, India

${ }^{2}$ Eros Paramount, Katraj, Pune, India

\begin{abstract}
A technique that could be used for sensing organic compounds is presented. In ethanol, Rhodamine-6G (a laser dye) was dissolved to obtain a Rhodamine-ethanol (Rh-Eth) solution with the concentration of Rhodamine-6G being $0.005 \mathrm{M}$. Methanol $\left(0.25 \mathrm{~cm}^{3}\right)$ was added to the Rh-Eth solution and the Rh-Eth-Me solution so obtained was pumped with a nitrogen laser. A blue shift in the fluorescence spectrum of the Rh-Eth-Me was observed as compared to the Rh-Eth solution without methanol. A similar blue shift was observed in the Rh-Eth solution when acetone and acetonitrile were separately added. When Rhodamine-6G and Coumarin 460 were added with Polymethyl methacrylate (PMMA) in ethanol, a blue shift was observed in the fluorescence spectrum as compared to the Rh-6G and Cou460 in ethanol without PMMA. The phenomena of spectral shifts in the fluorescence spectrum can be used to sense organic compounds and a mechanism is discussed.
\end{abstract}

Keywords: Nitrogen laser; Rhodamine-6G dye; Coumarin460 dye; Fluorescence; Organic compounds

\section{Introduction}

Attempts have been made to develop sensors and techniques that are capable of sensing a broad range of gases. The sensors and techniques are desired to be fast (in terms of response times), reversible, and highly sensitive. Rapid developments in the chemical analysis domain has forced for the development of efficient analytical sensors and techniques.

More specifically, attempts have been made to develop sensors and techniques based atomic and/or molecular properties. For example, the changes and/or modification in the electronic absorption and emission spectra of organic molecules in solvation processes can be used to develop sensors and techniques of interest. Attempts to evidence some empirical relations between the spectral characteristics and the microscopic or macroscopic parameters of the solutions were made in the first stage of research on the intermolecular interactions. Then, the interactions in the condensed phase were theoretically described in some kinetic and cell models [1-4]. Very large category of interactions act in solutions, so, the verification of the existent theories is made only in some restrictive situations, neglecting a series of contributions to the spectral shifts study of the effect of solvents on the electronic absorption and fluorescence spectra of a variety of molecules (e.g., azo compounds, azo disperse red dyes [5] forms an important subject for research in least years because can play a significant role in the photophysics of the excited states [6]. Some electro-optical parameters of the solute molecules can be estimated by spectral means, especially when the solute molecules are active both in absorption and in fluorescence electronic spectra. In solutions, the solvent environment determines important changes in the electro-optical properties of the spectrally active molecule compared to those in its gaseous phase. Several reports are now available on the correlation of UV absorption frequencies with the solvent parameters $[7,8]$. In the present paper a technique that could be used for sensing organic compounds is presented.

\section{Experimental Section}

In the present paper, ethanol was used to dissolve the laser dyes including coumarin-460 (Cou460), coumarin-450 (Cou450) and rhodamine-6G (Rh-6G). Each of the laser dyes, namely, coumarin-460
(Cou460), coumarin-450 (Cou450), and rhodamine-6G (Rh-6G), were dissolved separately in $50 \mathrm{~cm}^{3}$ of ethanol to obtain dye-ethanol (D-Et) solutions having molar concentration of $0.01 \mathrm{M}, 0.01 \mathrm{M}$, and $0.005 \mathrm{M}$, respectively. These particular concentrations were selected because the respective laser dyes exhibit lasing action at the afore-stated molar concentrations.

Once the dye-ethanol solutions were prepared, each of the solutions, one at a time, were placed in a quartz cuvette. The quartz cuvette used had four optically polished walls. The amount of solutions placed in the quartz cuvette was $1 \mathrm{~cm}^{3}$. Thereafter, a suitable amount of methanol (organic solvent) was added to the D-Et solutions to obtain dye-ethanol-methanol (D-Et-Me) solutions. Each of the D-Et-Me solutions so obtained were sonicated to obtain well mixed D-Et-Me solutions. The amount of methanol added, in each of the three D-Et solutions, was $0.25 \mathrm{~cm}^{3}$.

Each of the well mixed D-Et-Me solutions were further probed using the experimental set-up, a schematic of which is depicted in Figure 1, to obtain a fluorescence spectrum for each of the three D-EtMe solutions.

The experimental set-up comprised the following components (as shown in Figure 1 herein below):

A nitrogen laser (101) having a wavelength $(\lambda) 337.1 \mathrm{~nm}$, which was used to pump the laser dye in the D-Et-Me solutions. The quartz cuvette (103) (or the dye cell) for holding containing the D-Et-Me solution, which was placed in the path of the light incident from the

*Corresponding author: Jagdish W Dadge, Department of Physics, College of Engineering, Shivajinagar, Pune-411005, India, Tel: 020-25507501; E-mail: jwd.physics@coep.ac.in

Received September 20, 2017; Accepted October 06, 2017; Published October 25, 2017

Citation: Dadge JW, Thakur D (2017) A Technique for Sensing Organic Compounds Using Fluorescence Maximum Shift In Laser Dyes. J Laser Opt Photonics 4: 167. doi: 10.4172/2469-410X.1000167

Copyright: (C) 2017 Dadge JW, et al. This is an open-access article distributed under the terms of the Creative Commons Attribution License, which permits unrestricted use, distribution, and reproduction in any medium, provided the original author and source are credited. 


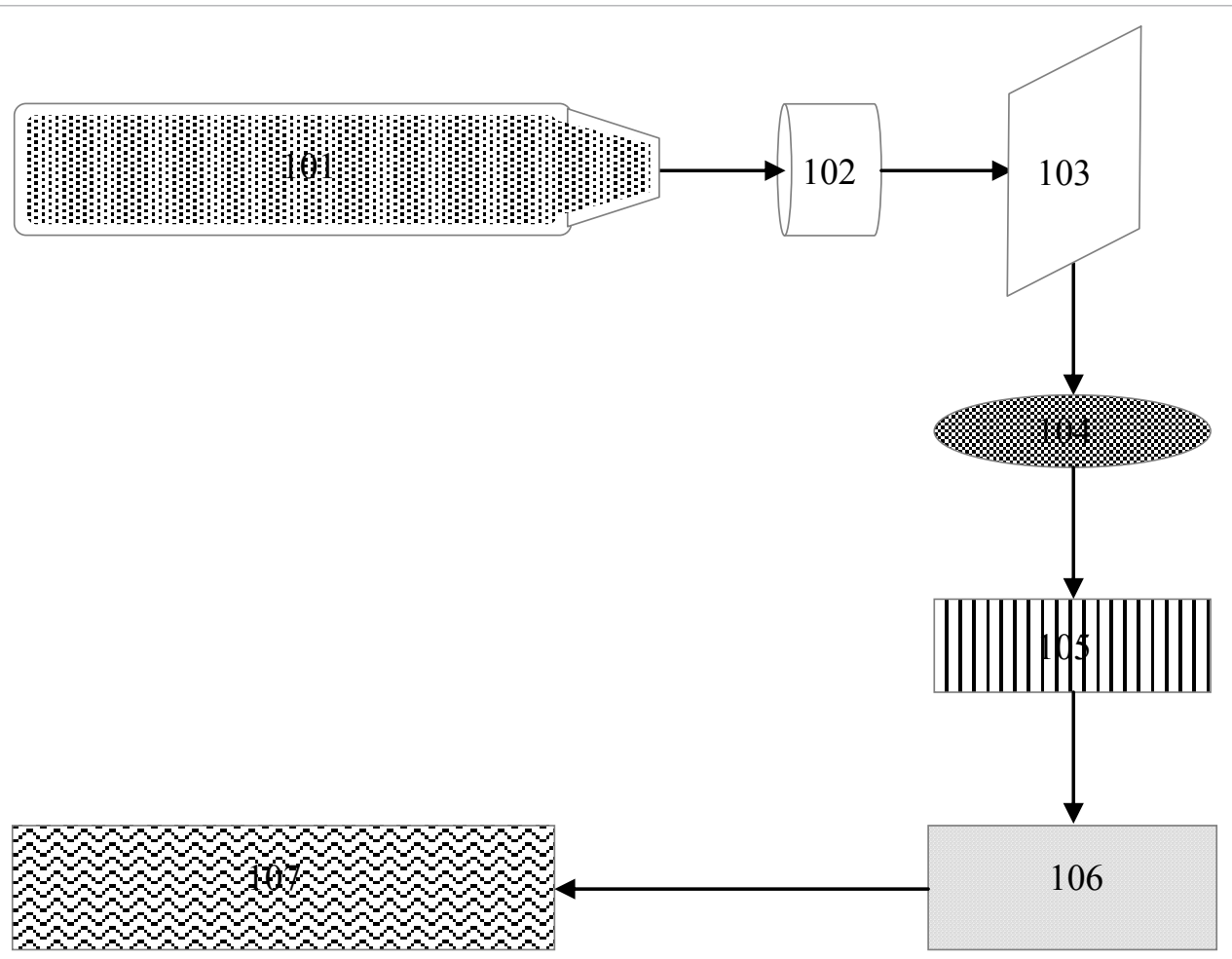

Figure 1: Experimental setup for recording the fluorescence spectra of the laser dyes dissolved in ethanol with and without organic solvents.

nitrogen laser. A cylindrical lens (102) disposed between the nitrogen laser (101) and the quartz cuvette (103), the cylindrical lens (102) was configured for collimating the nitrogen laser (101) on to the quartz cuvette (103).

Further, a monochromator (105) (Mc Pherson model 218 USA make, a least count of $0.05 \mathrm{~nm}$ ) was disposed orthogonal to the direction of incidence of the nitrogen laser. The monochromator (105) was configured to select desired wavelength from the fluorescence spectra obtained from the D-Et-Me solution (on excitation by the nitrogen laser). A quartz lens (104) (having a focal length of $14 \mathrm{~cm}$ ) was disposed between the quartz cuvette (103) and the monochromator (105) for focusing the light from the quartz lens (104) on to a slit of the monochromator (not shown in Figure 1). Beyond the monochromator (105), a photomultiplier tube (106) (EMI 9558) was placed for detecting/ amplifying and converting the light from the monochromator (105) into a digital signal.

The quartz lens (104), the monochromator (105), the photomultiplier tube (106) along with a recorder (107) formed the collecting optics. The recorder (107) used was x-t recorder from Omni scribe, Digital Electronics, and Bombay.

It is evident that any other laser source, other than nitrogen laser, can be used subject to the constraint that the light emitted by the laser source is absorbed by the dyes. The three dyes selected in the present study had fluorescence spectra in the visible range i.e., $400 \mathrm{~nm}$ to 650 nm.

Output of the nitrogen laser of 7 ns pulse duration (FWHM), 1 pulse per second was focused, with the cylindrical lens (having focal length of $8 \mathrm{~cm}$ ), on to the quartz cuvette containing the D-Et-Me solution. The dimensions of the quartz cuvette were $10 \mathrm{~mm} \times 10 \mathrm{~mm} \times$ $10 \mathrm{~mm}$ of Helm USA make and having a Teflon top cover. The quartz cuvette was mounted on a kinematic mount, which had means for adjusting the position of the quartz cuvette in both $\mathrm{x}$ and $\mathrm{y}$ directions with accuracy.

As described herein above with reference to the organic solvent methanol, other organic solvents including acetone and acetonitrile were added to the Rh-6G-Et solution. The amount of the organic solvents was $0.25 \mathrm{~cm}^{3}$, whereas the amount of the Rh-6G-Et dye solution was $1 \mathrm{~cm}^{3}$.

Further, polymethyl methacrylate (PMMA) admixed with Rh-6GEt dye solution along with chloroform and Cou460-ethanol admixed with PMMA along with Chloroform were also investigated using the experimental setup described herein above.

\section{Results and Discussion}

To start with a feasibility study was carried out, wherein methanol in an amount $0.25 \mathrm{~cm}^{3}$ was added to the dye-ethanol solution of each of the three laser dyes. The three laser dyes, Rh-6G, Cou460 and Cou450 were selected for the fluorescent detection of the organic solvents, on the basis of their high quantum yield in the organic solvents. Out of the three, Cou460-ethanol $(0.01 \mathrm{M})$ and Rh-6G-ethanol $(0.005 \mathrm{M})$ exhibited the strongest response to methanol, as compared with Cou450-ethanol $(0.01 \mathrm{M})$. On this basis, further studies were performed with Cou460ethanol and Rh-6G-ethanol.

The investigation results of each of the two dye-ethanol solutions (of Cou460 and Rh-6G), wherein the shift in the wavelength of the spectral maximum of the fluorescent spectrum on addition of the organic solvents, namely, methanol, acetonitrile, acetone, PMMA in chloroform to the two dye-ethanol solutions are listed in Table 1. In order to confirm the reproducibility of the results, the measurements 
were repeated at least twice and were found to be reproducible with the accuracy of $\pm 0.1 \mathrm{~nm}$ (Figures 2 and 3 ).

Figure 4 depicts a graph of variation in the fluorescence intensity (AU) versus wavelength for Rh-6G-ethanol (pure form) and $\mathrm{Rh}$ 6G-ethanol in different organic solvents, wherein the fluorescence wavelength maximum shift is clearly evident on comparing the fluorescence wavelength maximum of Rh-6G-ethanol with each of Rh-6G-ethanol-acetone, Rh-6G-ethanol-methanol, and Rh-6Gethanol-acetonitrile. Further, Figure 5 depicts a graph of variation in the fluorescence intensity (AU) versus wavelength for Rh-6G-ethanol and Rh-6G-ethanol in PMMA.

The shifts in the fluorescence wavelength maximum for Rh6G-ethanol (Figures 4 and 5) can be explicated with the aid of the chromospheres of the laser dye compounds. In order to explain the $\pi$-electron distribution in the chromophore of the xanthene dyes one has to take into consideration the two identical mesomeric structures

\begin{tabular}{|l|l|c|}
\hline S. NO. & Sample & $\boldsymbol{\lambda}_{\max }(\mathbf{n m})$ \\
\hline 1 & Rh-6G/ethanol & 585.4 \\
\hline 2 & Rh-6G/ethanol+Methanol & 584.2 \\
\hline 3 & Rh-6G/ethanol+Acetonitrile & 583.1 \\
\hline 4 & Rh-6G/ethanol+Acetone & 581.1 \\
\hline 5 & Rh-6G/ethanol+PMMA+Chloroform & 579.9 \\
\hline 6 & Cou460/ethanol & 457 \\
\hline 7 & Cou460/ethanol+PMMA+Chloroform & 453.6 \\
\hline
\end{tabular}

Table 1: Fluorescence wavelength maxima for Rh-6G/ethanol when organic solvents were introduced and PMMA dissolved in Chloroform were added to Rh6G/ethanol and Cou460/ethanol.<smiles>CCN(CC)c1ccc2c(C)cc(=O)oc2c1</smiles><smiles>CN(C)c1ccc2ccc(=O)oc2c1</smiles>

a: Molecular Structure of Coumarin 460<smiles>C[N+](C)=c1ccc2ccc([O-])oc-2c1</smiles>

c: Mesomeric form of Coumarin 460

Figure 2: Shows molecular structure and mesomeric forms of Cou460.

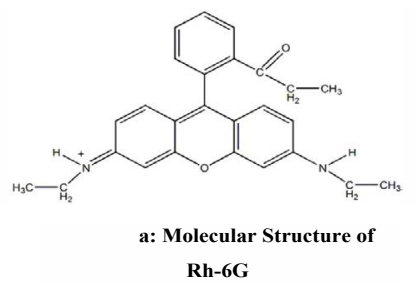

Rh-6G

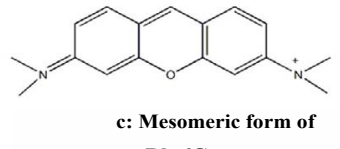

Rh-6G
Figure 3: Shows molecular structure and mesomeric forms of Rh-6G. of Rh-6G. Figure 3a depicts the molecular structure of Rh-6G, whereas Figure $3 b$ and Figure $3 c$ depicts the mesomeric structures of Rh-6G. In contrast to the coumarin dyes, the forms of Rh-6G depicted in Figure $3 \mathrm{~b}$ and Figure $3 \mathrm{c}$ appear to have the same weight. From the figures, it is evident that there is no static dipole moment that is parallel to the longitudinal axis of the xanthene dye molecule in both the ground and excited state. The transition moment of the main absorption band which arise in the wavelength ranging from $450 \mathrm{~nm}$ to $600 \mathrm{~nm}$ is oriented parallel to the longitudinal axis of the molecule. Few of the transitions at shorter wavelengths are oriented perpendicular to the longitudinal axis of the molecule [3].

Further, the absorption maximum is identified to be dependent on the solvent type in case of Rhodamine. More specifically, the absorption maximum is more marked effect in case of those dyes whose amine groups are not fully alkylated, such as Rh-6G and Rh-110 [4,5]. It is known that Rhodamine dyes which have a free (non-esterified) - $\mathrm{COOH}$ group can exist in several forms. Particularly, in polar solvents like ethanol or methanol, the carboxyl group plays a significant role in

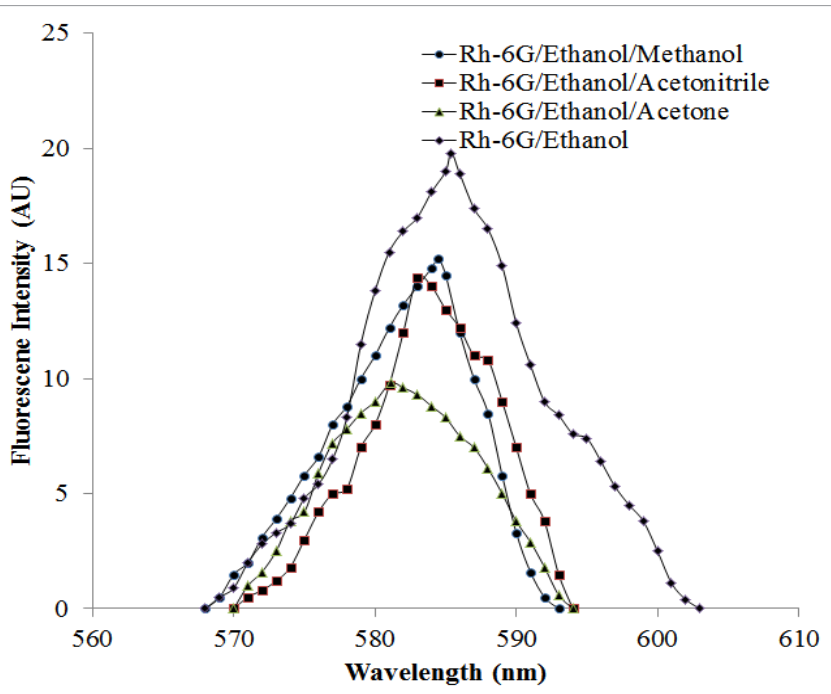

Figure 4: Fluorescence spectra Rh-6G/ethanol containing different solvents.

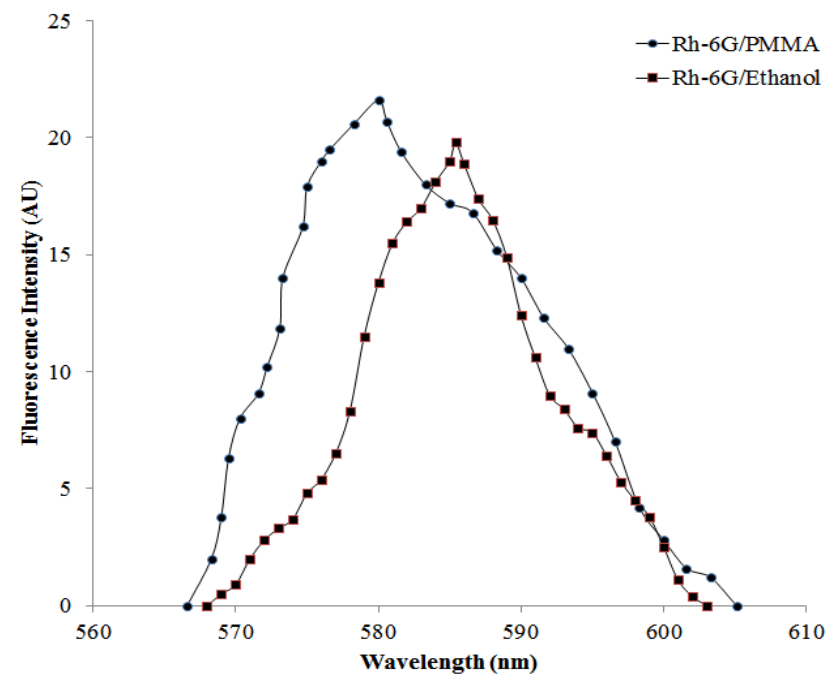

Figure 5: Fluorescence spectra of Rh-6G-ethanol in PMMA 


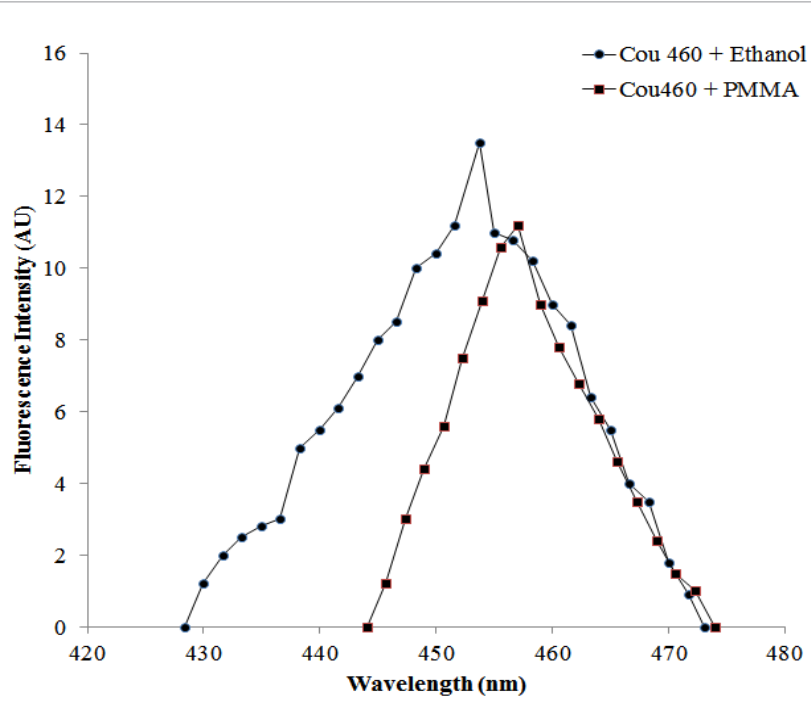

Figure 6: Fluorescence Spectra of Cou460/Ethanol in PMMA.

a typical acid-base equilibrium. The dissociation of Rhodamine dyes is further enhanced by dilution or by adding a small amount of a base. The zwitterionic forms of the dye are shifted to shorter wavelengths. This leads to shift of the fluorescence maximum towards shorter wavelengths i.e., towards the blue of the spectrum. Consequently, the dye solution (dye-ethanol) when added with the polar solvents like methanol and acetonitrile, the fluorescence maximum shifts towards blue side due to the zwitterionic form of the dye under consideration. On the other hand on addition of non-polar solvents like acetone, the zwitterionic form is not stable. Instead, an inner lactone is formed in a reversible reaction (Figure 5).

This is colorless since the $\pi$-electron system of the dye chromospheres is interrupted. In the presence of molecular oxygen a xanthene derivatives rapidly forms and indicates its strong blue fluorescence [6] (Figure 6).

Fluorescence spectra of Rh-6G-ethanol in PMMA matrix is depicted in Figure 5 and the fluorescence spectra of Cou460-ethanol in PMMA matrix is depicted in Figure 6. On comparison of the fluorescence quantum yield of Rh-6G and Cou460 in PMMA and that of Rh-6G and Cou 460 in ethanol, it was found that high fluorescence yield of Rh-6G and Cou460 occurred in PMMA. One rationale for this high fluorescence yield may be attribute to the rigidity of PMMA or high viscosity of PMMA. Because of the rigidity of PMMA or high viscosity of PMMA the rotational and vibrational relaxation of laser dye molecules is reduced and/or prevented, wherein the vibrational and rotational relaxation processes are the main reason for the radiative energy losses [6,7]. The non-radiative decay of the lowest excited singlet state $S_{1}$ directly to the ground state $S_{0}$ is generally responsible for the loss of fluorescence efficiency in organic dyes. Depending on the molecular structure of the dye and the properties of the solvent, the rate of relaxation can vary by many orders of magnitude.

There are several different structural features which contribute to the non-radiative decay $S_{1} \rightarrow S_{0}$. Several intra-molecular processes are responsible for quenching of the fluorescence. If a part of a dye molecule is strongly electron donating or with drawing reversible charge transfer may occur between this group and the excited chromophore resulting in the loss of electronic excitation [6]. Likewise a substituent with a low lying singlet or triplet state may quench the fluorescence via energy transfer.
It was observed that the fluorescence spectrum shifts towards the blue side of the spectrum when PMMA added to Cou460- ethanol, as depicted in Figure 6 and listed in Table 1. This can be explained by the chromophores of the Cou 460 dye compound; that is, mesomeric forms as depicted in Figure $2 b$ and $2 c$. This blue shift is mainly caused by the highly polar structure of the coumarin dye in the excited state, which shifts the absorption spectrum and hence the fluorescence spectra towards the blue side of the spectrum. Hydrogen bonding has been found to play an important role in the photophysics of the derivatives [6,8-10]. This type of bonding causes a blue shift in fluorescence.

Our approach to fluorescence-based chemical sensing is in some ways analogous to that of the dye/matrix film for organophosphonate vapors described previously by Grate $[1,11]$.

\section{Conclusion}

The results discussed above can help to determine the feasibility of employing temporal response information. It is felt that the temporal response information may be beneficial for improving the maximum shift of the sensor and for analyzing all polar and non-polar solvents.

The laser dye Rh-6G-ethanol when dissolved in different organic solvents and PMMA can be used to detect simple organic compounds via the fluorescence of the dye upon excitation with a nitrogen laser at $337.1 \mathrm{~nm}$ as is shown in the present study.

Particularly, the combination of Rh-6G-ethanol-acetone showed the maximum blue shift as compared to Rh-6G-ethanol-acetonitrile and Rh-6G-ethanol-methanol. Further, the fluorescence of the Rh6G-ethanol-PMMA and Cou460-ethanol-PMMA showed blue shift as compared to Rh-6G-ethanol and Cou460-ethanol respectively. The results can be attributed to the laser-induced variations in physical properties of the laser dye upon interaction with the organic solvents or polymer support for the sensors.

\section{References}

1. Grate JW, Levitsky I, Krivoshlykov SG (2001) Rational design of a Nile red/ polymer composite film for fluorescence sensing of organophosphate vapours using hydrogen bond acidic polymers. Ana Chem 73: 3441-3448.

2. Mark J Muldoon, Charles M Gordon, Ian R Dunkin, (2001) Investigations of solvent solute interactions in room temperature ionic liquids using solvatochromic dyes. J Chem Soc Perkin Trans 2 433-435.

3. Jakobi H, Kuhn H (1962) Z Elektrochem. Ber. Bunsenges. Phys Chem 66: 46.

4. Drexhage KH (1972) Design of laser dyes, VII International Quantum Electronics Conference Montreal, Canada.

5. Drexhage KH (1973) Laser Focus 9: 35.

6. Schafer FP (1990) Dye lasers. Springer, Verlag, Berlin, pp: 161-179.

7. Jones G, Jackson WR, choi CY, Bergmark WR, (1985) Solvent effects on emission yield and lifetime for coumarin laser dyes: Requirements for a rotatory decay mechanism. J Phys Chem 89: 294.

8. AV Deshpande EB Namdas (1997) Lasing action of rhodamine B in polyacrylic acid films. Appl Phys B 64: 419.

9. Li D, Mills CA and Cooper JM. (2003) Microsystems for optical gas sensing incorporating the solvatochromic dye Nile red. Sensors and Actuators B: Chemical 92: 73-78.

10. Watt DR (2002) Array analysis SPIE's Oemagazine of Photonics Technologies and Applications 220-22.

11. Machado, Clodoaldo, Machado, Vanderlei Grayer (2001) J Chem.Edu 78: 649 